\title{
Adherencia terapéutica de pacientes con hipertensión arterial y diabetes mellitus tipo 2 de Bucaramanga, Colombia
}

\author{
Adherence to therapeutic regimen of hypertension and \\ type-2 diabetes patients in Bucaramanga, Colombia
}

Sandra L. Romero G. ${ }^{1}$, Dora I. Parra ${ }^{1}$, Javier M. Sánchez R. ${ }^{1}$, Lyda Z. Rojas ${ }^{1}$

Forma de citar: Romero S, Parra D, Sánchez J, Rojas L. Adherencia terapéutica de pacientes con hipertensión arterial y diabetes mellitus tipo 2 . Rev Univ Ind Santander Salud. 2017; 49(1): 37-44. DOI: http://dx.doi.org/10.18273/revsal.v49n1-2017004 (c) (1)

\section{RESUMEN}

Introducción: Existen múltiples métodos o instrumentos para medir adherencia, la mayoría de ellos enfocados en la toma de medicamentos. Sin embargo, también es importante evaluar otros aspectos relacionados con el cumplimiento del régimen terapéutico en pacientes con hipertensión y diabetes mellitus como, la dieta, el ejercicio y otras recomendaciones dadas por los profesionales de la salud para controlar la enfermedad y evitar su progreso. Objetivo: Determinar y describir el nivel de adherencia al régimen terapéutico en pacientes con hipertensión arterial o diabetes mellitus tipo 2. Métodos: Estudio de corte transversal analítico, realizado en pacientes de dos programas de riesgo cardiovascular. La adherencia fue medida con la etiqueta de resultado de enfermería "Conducta terapéutica: enfermedad o lesión (1609)". Se llevó a cabo un análisis descriptivo del nivel de adherencia y adicionalmente analítico estratificando por edad, sexo y enfermedad que padece. Resultados: Se incluyeron 500 pacientes para este estudio. La mediana de edad fue de 68 años y el $69.0 \%$ eran mujeres. El promedio general de adherencia fue de $3.11 \pm 0.4$ puntos. Cinco de doce indicadores se ubicaron en las categorías de nunca y raramente demostrado. No se encontraron diferencias estadísticamente significativas al estratificar edad, sexo y enfermedad $(\mathrm{p}=0.629, \mathrm{p}=0.396 \mathrm{y} \mathrm{p}=0.535$, respectivamente). Conclusiones: Se estableció un promedio general de la adherencia al régimen terapéutico de a veces demostrada, con cinco indicadores bastante comprometidos. Evaluar el nivel adherencia con este instrumento permite identificar las áreas de menor cumplimento con el propósito que el personal de enfermería pueda orientar su plan de cuidados.

Palabras clave: Cumplimiento de la medicación, hipertensión, diabetes mellitus tipo 2, evaluación en enfermería, prevención secundaria.

\footnotetext{
ABSTRACT

Introduction: Several methods or instruments exist for measuring adherence, and most of them focused on medication intake. However, it is also important to assess other aspects related to compliance with therapeutic

1. Universidad Industrial de Santander. Bucaramanga, Colombia.

Correspondencia: Sandra L. Romero G. Dirección: Carrera 32 No 29-31 Escuela de Enfermería Universidad Industrial de Santander, Teléfono: (7) 6344000 Ext: 3152-3009, Correo electrónico: salurome@uis.edu.co 
regimen in hypertension and type-2 diabetes patients, such as diet, exercise, and other recommendations by health professionals to control disease and avoid its progress. Objective: To determine and describe level of adherence to therapeutic regimen in hypertension and type-2 diabetes patients. Methods: Cross-sectional descriptive study conducted in patients of two cardiovascular risk programs. Adherence was measured through the nursing outcomes label "Therapeutic behavior: illness or injury (1609)". A descriptive analysis of adherence level, including stratifications by age, sex and disease, was carried out. Results: A total 500 patients were included. Median age was 68 years and $69.0 \%$ were women. General mean adherence level was $3.11 \pm 0.4$ points. Five of twelve indicators were among the never-and-rarely-demonstrated categories. No statistically significant differences were found when stratifying age, sex and disease ( $\mathrm{p}$-value $=0.629, \mathrm{p}$-value $=0.396$ and $\mathrm{p}$-value $=0.535$, respectively). Conclusions: A general average level of adherence to therapeutic regimen in the category sometimes demonstrated was established with five indicators showing high compromise levels. Evaluation of adherence level with this instrument allows identification of areas with low compliance so that professional nursing staff can guide their care plans.

Keywords: Medication adherence, patient compliance, hypertension, Diabetes mellitus type 2, nursing assessment, secondary prevention.

\section{INTRODUCCIÓN}

La hipertensión arterial (HTA) y la diabetes mellitus tipo 2 (DM) son enfermedades crónicas no transmisibles (ECNT), que generan una alta carga de morbimortalidalidad por enfermedades cardiovasculares ${ }^{1}$. Según informe de 2014 de la Organización Mundial de la Salud (OMS), la situación mundial de las ECNT siguen siendo la principal causa de muerte en el mundo, responsables del $68 \%$ de muertes de los 56 millones de defunciones registradas en el 2012. Adicionalmente, 16 millones de ellas (40\%) fueron muertes prematuras ocurridas antes de los 70 años de edad ${ }^{2}$. Bajo este contexto, se prevé que tanto la HTA como la DM y sus complicaciones serán las principales amenazas de los recursos de la salud pública en todo el mundo, con un enorme costo económico y social $^{1,3}$.

Pese a las distintas opciones terapéuticas disponibles en la actualidad para el control de la enfermedad, el 33-49\% de los pacientes aún no cumplen las metas terapéuticas de glucemia, presión arterial o control del colesterol y sólo el 14\% logran los objetivos para las tres medidas ${ }^{4,5}$. Es así, que la prevalencia de adherencia a la medicación en pacientes con HTA oscila entre $52 \%$ y $74 \%{ }^{6}$ y en personas con DM entre el $36 \%$ al $93 \%$. Este hecho ha llevado a la generación de diversos estudios con el propósito de evaluar la adherencia durante el proceso de atención ${ }^{6-8}$.

Existen múltiples métodos o instrumentos para medir la adherencia ya sea de forma directa o indirecta ${ }^{9-11}$, la mayoría de ellos enfocados en la toma de medicamentos. Con el propósito de evaluar la adherencia desde un enfoque integral que incluya, tanto lo farmacológico como lo no farmacológico tales como la dieta, el ejercicio y otras recomendaciones dadas por los profesionales de la salud para el control de la enfermedad, se realizó este estudio con el objetivo determinar y describir el nivel de adherencia al régimen terapéutico en pacientes con hipertensión arterial o diabetes mellitus tipo 2, y adicionalmente establecer el nivel de adherencia estratificando por edad, sexo y enfermedad que padece.

\section{MATERIALES Y MÉTODOS}

Estudio de corte transversal analítico en pacientes con diagnóstico médico de HTA o DM tipo 2 pertenecientes a programas de riesgo cardiovascular de dos instituciones de atención primaria (pública y privada), realizado en el periodo comprendido entre el 15 de enero y 15 mayo de 2013 en la ciudad de Bucaramanga-Colombia.

Se incluyeron pacientes mayores de 18 años, totalmente independientes de su cuidado y asistentes activos a los programas de riesgo cardiovascular. Se consideraron activas todas aquellas personas que asistieron como mínimo a un control en los últimos seis meses, esto con el propósito de facilitar su ubicación y contacto. Fueron excluidas mujeres embarazadas y aquellas personas con alteración en la esfera mental, con alteraciones crónicas o graves y con limitaciones de la comunicación que le impedían responder la entrevista.

Se calculó un tamaño de muestra teniendo en cuenta una población disponible de 7.000 personas, una adherencia esperada al régimen terapéutico del $50 \%$ de acuerdo a parámetros de la $\mathrm{OMS}^{12}$, una confianza del $95 \%$, un efecto de diseño de 1 y pérdidas por tasa de no respuesta del $20 \%$, resultando en un tamaño maestral del 438 personas. Finalmente, con el equipo investigador se 
decidió tomar un total de 500 pacientes, con el fin de contar con un poder adecuado, considerando que el estudio tenía otros objetivos adicionales. La selección de la muestra se realizó de forma probabilística, a través de un muestreo aleatorio simple, utilizando números aleatorios generados en un software estadístico.

Una enfermera profesional fue la encargada de recolectar la información, quien con el listado de pacientes seleccionados aleatoriamente, los contactaba telefónicamente o mediante una visita domiciliaria. Una vez el paciente manifestaba su interés de participar en el estudio, se acordaba una cita para obtener el consentimiento informado por escrito y poder llevar a cabo la entrevista, la cual tenía una duración de aproximadamente 25 a 30 minutos. Si el paciente no deseaba participar, se tomaba la siguiente persona del listado.

El formato de recolección de datos contempló aspectos como: las características sociodemográficas, clínicas y la adherencia al régimen terapéutico. Esta última medida con la etiqueta de Clasificación de Resultados de Enfermería (Nursing Outcomes Classification) Conducta terapéutica: enfermedad o lesión ${ }^{13}$ definida como las "acciones personales para paliar o eliminar patología", previamente validada en personas con HTA o DM, la cual cuenta con la validez de constructo realizada a través del análisis Rasch y la evaluación de la reproducibilidad prueba-reprueba donde se encontró un coeficiente de correlación intraclase de $0.70^{14}$. Esta etiqueta contempla 12 indicadores operacionalizados en 11 categorías, que contienen 85 ítems dicótomos que evalúan el cumplimiento al régimen terapéutico, medidos con una escala tipo likert de 1 a 5 puntos, donde 1 corresponde a nunca demostrado, 2=Raramente demostrado, $3=\mathrm{A}$ veces demostrado, $4=$ Frecuentemente demostrado y $5=$ Siempre demostrado ${ }^{13}$.

Se realizó un análisis descriptivo de las características de la población estudio y de la adherencia al régimen terapéutico. Las variables en escala de medición cualitativa se describen las frecuencias absolutas y relativas, las variables continuas que presentaron una distribución normal en la prueba Shapiro Wilk se reportó la media y desviación estándar, de lo contrario se informó la mediana y primer y tercer cuartil (Q1Q3). Usamos pruebas T-Student y ANOVA para identificar diferencias al estratificar por edad, sexo y enfermedad. Se consideró diferencias estadísticamente significativas con una valor de $\mathrm{p}<0.05$. Se utilizó el software estadístico Stata versión 14 para el análisis.

\section{RESULTADOS}

Un total de 500 pacientes fueron estudiados. La mediana de edad de la población fue de 68 años (Q1=59 años; Q3=75 años), en cuanto a sus características sociodemográficas, el $69.0 \%$ eran mujeres, el $99.4 \%$ pertenecían al estrato socioeconómico bajo y medio, el $75.4 \%$ eran analfabetas o solo contaban con educación primaria, otras características sociodemográficas se pueden observar en la TABLA 1.

TABLA 1. Características sociodemográficas de pacientes con hipertensión arterial o diabetes mellitus tipo $2(\mathrm{n}=500)$.

\begin{tabular}{|c|c|}
\hline Variable & n (\%) \\
\hline \multicolumn{2}{|l|}{ Sexo } \\
\hline Mujer & $345(69.0)$ \\
\hline Hombre & $155(31.0)$ \\
\hline \multicolumn{2}{|l|}{ Estado civil } \\
\hline Soltero & $85(17.0)$ \\
\hline Casado/Unión libre & $261(52.2)$ \\
\hline Divorciado/Separado & $34(6.8)$ \\
\hline Viudo & $120(24.0)$ \\
\hline \multicolumn{2}{|l|}{ Estrato } \\
\hline Bajo & $290(58.0)$ \\
\hline Medio & $207(41.4)$ \\
\hline Alto & $3(0.6)$ \\
\hline \multicolumn{2}{|l|}{ Nivel educativo } \\
\hline Analfabeta & $55(11.0)$ \\
\hline Primaria & $312(65.4)$ \\
\hline Secundaria & $110(22.0)$ \\
\hline Técnico & $17(3.4)$ \\
\hline Universitarios/postgrados & $6(1.2)$ \\
\hline \multicolumn{2}{|l|}{ Ocupación } \\
\hline Empleado & $33(6.6)$ \\
\hline Independiente & $75(15.0)$ \\
\hline Desempleado/Cesante & $74(14.8)$ \\
\hline Pensionado/Jubilado & $94(18.8)$ \\
\hline Hogar/Ama de casa & $224(44.8)$ \\
\hline \multicolumn{2}{|c|}{ Institución de salud para la atención primaria } \\
\hline Pública & $244(48.8)$ \\
\hline Privada & $256(51.2)$ \\
\hline
\end{tabular}

Fuente: Autores. 
En cuanto a las enfermedades de interés de la población estudiada, el $69.4 \%$ tenía HTA, el $9.0 \%$ DM y el $21.6 \%$ las dos patologías. El 90.0\% tenía ausencia de comorbilidad según el índice de Charlson ${ }^{15} 12 \%$ (181) y el $48.47 \%$ tenía un riesgo alto y muy alto de enfermedad cardiovascular a 10 años según Framingham ${ }^{16}$. En relación con el número de medicamentos, la mediana de pastillas o presentaciones farmacéuticas que tomaba el paciente al día fue de cinco pastillas $(\mathrm{Q} 1=3 ; \mathrm{Q} 3=7$ pastillas), en la Tabla 2 se puede apreciar el tratamiento farmacológico recibido.

TABLA 2. Características clínicas de pacientes con hipertensión arterial o diabetes mellitus tipo $2(n=500)$.

\begin{tabular}{|c|c|}
\hline Variable & n (\%) \\
\hline \multicolumn{2}{|l|}{ Patología } \\
\hline Hipertensión arterial & $347(69.4)$ \\
\hline Diabetes mellitus tipo 2 & $45(9.0)$ \\
\hline Hipertensión arterial y diabetes mellitus tipo 2 & $108(21.6)$ \\
\hline \multicolumn{2}{|l|}{ Hospitalizaciones en el último año } \\
\hline $\mathrm{Si}$ & $56(11.24)$ \\
\hline No & $442(88.76)$ \\
\hline \multicolumn{2}{|l|}{ Índice de Charlson } \\
\hline Ausencia de comorbilidad & $450(90.0)$ \\
\hline Baja comorbilidad & $41(8.2)$ \\
\hline Alta comorbilidad & $9(1.8)$ \\
\hline \multicolumn{2}{|l|}{$\begin{array}{l}\text { Riesgo de enfermedad general cardiovascular } \\
\text { a } 10 \text { años (Framingham) }\end{array}$} \\
\hline Bajo & $186(38.0)$ \\
\hline Moderado & $67(14.0)$ \\
\hline Alto & $102(20.0)$ \\
\hline Muy alto & $136(28.0)$ \\
\hline \multicolumn{2}{|l|}{ Tratamiento farmacológico } \\
\hline $\begin{array}{l}\text { Inhibidores de la enzima convertidor de } \\
\text { angiotensina }\end{array}$ & $133(26.6)$ \\
\hline Betabloqueadores & $130(26.0)$ \\
\hline Antagonistas de los receptores de angiotensina II & $259(51.8)$ \\
\hline Calcioantagonistas & $156(31.2)$ \\
\hline Diuréticos & $171(34.2)$ \\
\hline Hipolipemiantes & $241(48.2)$ \\
\hline Hipoglucemiantes & $142(28.4)$ \\
\hline Antiagregantes plaquetarios & $290(58.0)$ \\
\hline
\end{tabular}

Fuente: Autores.
En relación con la adherencia al régimen terapéutico el promedio general fue de $3.11 \pm 0.4$ puntos, indicando una adherencia de a veces demostrada. En cuanto a los indicadores, solo cuatro obtuvieron una media de calificación superior a 4.0, lo cual corresponde a frecuentemente adherente, entre ellos: cumple con el régimen de medicación (media 4.43 \pm 1.1 ), este indicador evalúa las indicaciones en la toma de medicamentos como la dosis, toma y horas indicadas; equilibra actividad y reposo (media $4.42 \pm 0.7$ puntos) que indagó aspectos como las horas de sueño, las actividades de recreación y descanso; sigue la dieta prescrita (media $4.09 \pm 0.9$ puntos) valora el cumplimento de una dieta baja en sal, grasas, azucares, harinas e incremento de consumo de frutas y verduras; evita conductas que potencian la patología (media $4.02 \pm 0.8$ puntos) que evalúa hábitos y situaciones que influyen en la presión arterial y diabetes como el consumo de alcohol y tabaco, así como situaciones que generan disgusto o preocupación.

En un nivel de a veces demostrado, se encontraron dos ítems que evalúan aspectos como modificar o interrumpir actividades del hogar o trabajo para cumplir con el tratamiento y la asistencia a citas o controles con un profesional de salud por diversos motivos. El primero corresponde a alterar las funciones del rol para el cumplimiento terapéutico (media $3.62 \pm 1.1$ puntos) y el segundo incluye los indicadores de solicitar cita con el profesional sanitario cuando es necesario y obtener asesoramiento de un profesional cuando es necesario (media $3.91 \pm 0.7$ puntos).

De otra parte, tres indicadores se ubicaron en el rango de raramente adherente, entre ellos: supervisa los efectos terapéuticos (media $2.65 \pm 1.1$ puntos), el cual evalúa la autosupervisión de la presión arterial o glucosa y control de peso; cumple el nivel de actividades prescritas (media 2.34 \pm 1.5 ) que indaga aspectos relacionados con la actividad física y supervisa los cambios en el estado de enfermedad (media 2.26 \pm 0.9 puntos) que valora el conocimiento de los síntomas de elevación de la presión arterial o glucosa.

Finalmente, las calificaciones más bajas de nunca adherente, fueron para los indicadores que evalúan aspectos relacionados con el conocimiento de las molestias generadas por el tratamiento farmacológico y el conocimiento de los síntomas que pueden estar indicando una complicación de ser diabético o hipertenso, entre ellos: supervisa los efectos secundarios de la enfermedad (media $1.23 \pm 0.5$ puntos) y del tratamiento (media $1.19 \pm 0.4$ puntos) Tabla 3. 
Adherencia terapéutica de pacientes con hipertensión arterial y diabetes mellitus tipo 2 de Bucaramanga, Colombia

TABLA 3. Nivel de adherencia al régimen terapéutico en pacientes con hipertensión arterial o diabetes mellitus tipo 2 ( $\mathrm{n}=500$ ).

\begin{tabular}{llc}
\hline Código & \multicolumn{1}{c}{ Conducta terapéutica: enfermedad o lesión (1609) } & Media/DE \\
\hline $\mathbf{1 6 0 9 0 5}$ & Cumple con el régimen de medicación & $4.43 \pm 1.1$ \\
$\mathbf{1 6 0 9 0 4}$ & Cumple el nivel de actividades prescritas & $2.34 \pm 1.5$ \\
$\mathbf{1 6 0 9 1 9}$ & Sigue la dieta prescrita & $4.09 \pm 0.9$ \\
$\mathbf{1 6 0 9 0 6}$ & Evita conductas que potencien la patología & $4.02 \pm 0.8$ \\
$\mathbf{1 6 0 9 1 6}$ & Solicita cita con profesional sanitario cuando es necesario & $3.91 \pm 0.7$ \\
$\mathbf{1 6 0 9 2 1}$ & Obtiene asesoramiento de un profesional cuando es necesario & $2.65 \pm 1.1$ \\
$\mathbf{1 6 0 9 0 8}$ & Supervisa los efectos terapéuticos & $2.26 \pm 0.9$ \\
$\mathbf{1 6 0 9 1 1}$ & Supervisa los cambios en el estado de enfermedad & $1.19 \pm 0.4$ \\
$\mathbf{1 6 0 9 0 9}$ & Supervisa los efectos secundarios del tratamiento & $1.23 \pm 0.5$ \\
$\mathbf{1 6 0 9 1 0}$ & Supervisa los efectos secundarios de la enfermedad & $3.62 \pm 1.1$ \\
$\mathbf{1 6 0 9 1 3}$ & Altera las funciones el rol para el cumplimiento terapéutico & $4.42 \pm 0.7$ \\
$\mathbf{1 6 0 9 2 0}$ & Equilibra actividad y reposo & $\mathbf{3 . 1 1} \pm \mathbf{0 . 4}$ \\
\hline Adherencia general &
\end{tabular}

DE=Desviación estándar

Fuente: Autores.

En cuanto a las características sociodemográficas y el proceso de enfermedad, no se encontraron diferencias estadísticamente significativas entre la media del nivel de adherencia al estratificar por edad, sexo y enfermedad. La media para el grupo etario de 35 a 59 años fue $3.08 \pm 0.38$, de 60 a 68 años $3.11 \pm 0.40$, de 69 a 75 años $3.13 \pm 0.31$ y de 76 a 92 años $3.08 \pm 0.31$, con un valor de $p=0.629$. La media de adherencia para las mujeres fue de $3.09 \pm 0.35$ vs $3.12 \pm 0.36$ para los hombres, con una $\mathrm{p}=0.396$. En cuanto a la patología, la media de adherencia para personas con HTA fue de $3.11 \pm 0.35$, para DM $3.06 \pm 0.39$ y para las personas con las dos patologías de $3.08 \pm 0.35$, con un valor de $\mathrm{p}=0.535$. Figura 1 .
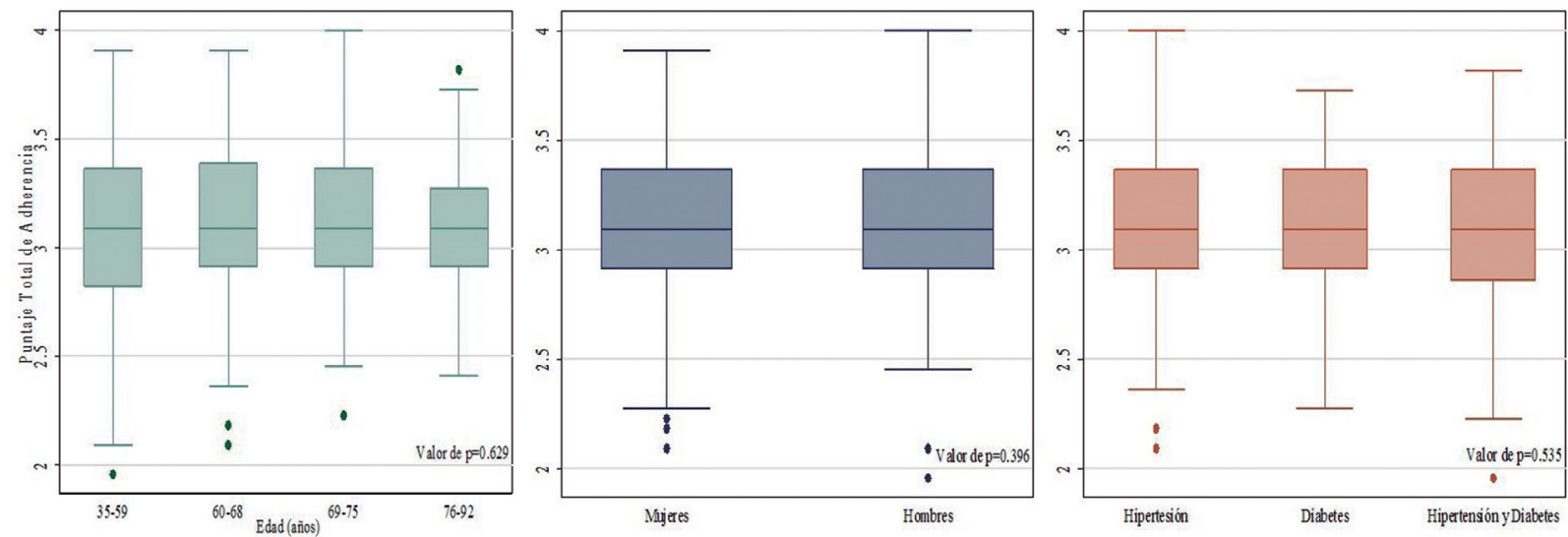

FIGURA 1. Nivel de adherencia al régimen terapéutico en pacientes con hipertensión arterial y diabetes mellitus según edad, sexo y enfermedad $(n=500)$.

Fuente: Autores.

\section{DISCUSIÓN}

Dentro de los principales hallazgos de este estudio se encuentra la caracterización de la adherencia al régimen terapéutico más allá de lo farmacológico, evaluando el cumplimiento de 12 importantes aspectos para el control de la enfermedad en pacientes con HTA y DM.
Esta investigación permitió identificar aspectos en los cuales los pacientes no son adherentes (supervisa los efectos secundarios de la enfermedad y del tratamiento), raramente adherentes (supervisa los efectos terapéuticos, cumple el nivel de actividades prescritas y supervisa los cambios en el estado de enfermedad) y a su vez, aspectos en los que los pacientes son frecuentemente 
adherentes (cumple con el régimen de medicación y equilibra actividad y reposo).

Por otra parte, la comparabilidad de nuestros hallazgos con otros estudios se hace difícil, debido a que el instrumento utilizado para medir la adherencia no ha sido empleado aun en otras investigaciones. A nivel mundial se han empleado diversos métodos directos e indirectos para medir el cumplimento al régimen terapéutico, la mayoría de ellos centrados en el tratamiento farmacológico y expresados en términos de proporciones. Por lo tanto, para facilitar la comparabilidad con otros estudios, se adaptaron los resultados a una escala porcentual.

En consideración con lo anterior, al puntaje general de adherencia al régimen terapéutico en los pacientes con HTA fue de $62.2 \%$ (media de 3.11 puntos) y del $61.2 \%$ en los diabéticos (media de 3.06 puntos). Estos valores se encuentran dentro del rango de resultados previamente descritos en la literatura, donde la adherencia a la medicación en pacientes hipertensos oscila entre el $52 \%$ y el $74 \%{ }^{6}$ y para los diabéticos entre el $36 \%$ y el $93 \%$. La variedad en estos resultados podría ser explicada por los múltiple métodos empleados para medir la adherencia ${ }^{11,17}$ y por el enfoque principalmente centrado en el tratamiento farmacológico ${ }^{4,9,10}$.

El indicador que evalúa el cumplimiento del tratamiento farmacológico "cumple con el régimen de medicación" fue el de mayor puntaje (media 4.43 puntos, equivalente al $88.6 \%$ ), lo cual coincide con varios estudios donde se ha reportado una alta adherencia a los medicamentos ${ }^{9,17,18}$. Este alto porcentaje podría estar sobreestimado por tratarse de un autoreporte ${ }^{19}$, donde el paciente generalmente tiene conocimiento sobre la importancia de tomar los medicamentos para su tratamiento o puede ser producto del sesgo de deseabilidad social.

En relación al indicador de cumplimiento de la actividad prescrita se encontró una baja adherencia (media 2.34 puntos, equivalente al $46.8 \%$ ), sin embargo, esta es superior a lo reportado en otros estudios como el de Thinyane, et al. ${ }^{20}$, quienes hallaron una adherencia al ejercicio de 7.1\% y el de Migneault, et al. ${ }^{21}$ quienes en la linea base de su ensayo clínico encontraron valores de $38.5 \%$ y $26.2 \%$ en la actividad física. Con respecto al indicador de dieta prescrita, el puntaje obtenido en nuestro estudio fue alto (media 4.09 puntos, equivalente al $81.8 \%$ ) comparado con otros estudios como el de Thinyane, et al. ${ }^{20} \mathrm{y}$ el de Migneault, et al. ${ }^{21}$ quienes encontraron valores de $37.1 \%$ y de $55.8 \%$ respectivamente.
Los aspectos mencionados en el párrafo anterior, dejan ver que los cambios en la conducta para generar estilos de vida saludables en personas con HTA o DM, siguen siendo uno de los principales retos para lograr la adherencia al régimen terapéutico en poblaciones con enfermedades crónicas, como la hipertensión y la diabetes $^{2,4,8,22,23}$.

Respecto a los indicadores relacionados con la supervisión que deben hacer los pacientes sobre los efectos terapéuticos, los cambios en el estado de la enfermedad, los efectos secundarios del tratamiento y los efectos secundarios de la enfermedad, no se encontraron estudios que evalúen estos aspectos, lo cual puede ser explicado por la poca educación brindada a los pacientes y el bajo conocimiento respecto a estos temas en personas con HTA o $\mathrm{DM}^{24}$. Por otra parte, se ha documentado que aquellas personas con mayor conocimiento sobre su propia salud, tienen mayor adherencia a las recomendaciones dadas por los profesionales de la salud, lo que a futuro representa un mayor control de las complicaciones ${ }^{25-29}$.

En relación con la edad y el sexo, en este estudio no se encontraron diferencias en el nivel de adherencia al estratificar por estas condiciones, a diferencia de lo reportado en la literatura en donde se refiere que los hombres con menor edad y menor tiempo con la enfermedad tienden a tener una menor adherencia; algunos destacan que por cada 10 años con el proceso de enfermedad la adherencia aumenta, siendo los 60 años o más donde hay mejor cumplimiento ${ }^{29-33}$. A pesar de los resultados de nuestro estudio, consideramos que estas variables deben ser tenidas en cuenta al momento de brindar educación y de implementar estrategias para aumentar la adherencia en este tipo de población.

Dentro de las fortalezas de este estudio resaltamos la medición de aspectos más allá del abordaje farmacológico del régimen terapéutico en personas con HTA y DM tipo 2, incluyendo indicadores relacionados con la dieta, ejercicio, control de conductas que potencian la patología, supervisión del estado de enfermedad, efectos terapéuticos entre otros. También, esta investigación contó con un cálculo de tamaño de muestra seleccionada de forma probabilística, haciéndola representativa de la población estudio. Otro aspecto a destacar es el instrumento utilizado para evaluar la adherencia, al cual se le realizó validación facial, de constructo y reproducibilidad por los mismos autores de este estudio, resultados que se encuentra en proceso de publicación. De otra parte, dicho instrumento hace parte del lenguaje estandarizado en enfermería. 
Una posible limitación de estudio fue haber considerado solo pacientes activos en los programas de riesgo cardiovascular, hecho que podría representar una población con mayor adherencia con respecto a los no activos, sin embargo, la captación y participación de los no asistentes hubiese significado mayor complejidad y riesgo para el cumplimiento de los objetivos del estudio. Otra posible limitación es el autoreporte que puede sobreestimar el cumplimiento, sin embargo, consideramos que otros métodos más objetivos no son prácticos ni económicos tanto para el paciente como para el personal de salud y no facilitan su uso en programas de riesgo cardiovascular en países en vía de desarrollo y en poblaciones con un bajo nivel educativo.

En relación con las implicaciones de este estudio para la práctica, el uso de este instrumento en conjunto con otros indicadores de control de la enfermedad, como lo es la medición de presión arterial, hemoglobina glucosilada y medidas antropométricas, entre otras, podría acércanos un poco más a ese constructo que denominamos adherencia.

En conclusión, este estudio evaluó la adherencia con un enfoque que supera lo farmacológico, encontrando un promedio general de adherencia al régimen terapéutico de a veces demostrada y un nivel de cumplimento bastante comprometido en cinco indicadores. Lo anterior permite a los profesionales de salud y en particular a enfermería, identificar las necesidades de educación y los aspectos en los que requieren ser apoyadas las personas con HTA y DM tipo 2, para un adecuado control de la enfermedad.

\section{AGRADECIMIENTOS}

Este proyecto hace parte de la Unión Temporal CARDIECOL, una alianza de varias instituciones educativas y de salud entre ellas la Universidad Industrial de Santander. Financiado por COLCIENCIASCARDIECOL convocatoria No 998-2014, código 47755.

\section{CONSIDERACIONES ÉTICAS}

Este estudio contó con el aval de los Comités de Ética en Investigaciones de las instituciones participantes. Todos los pacientes dieron el consentimiento informado por escrito.

\section{CONFLICTO DE INTERESES}

Los autores declaramos no tener conflictos de intereses.

\section{REFERENCIAS}

1. OMS. Informacióngeneral sobrelaHIPERTENSIÓN en el mundo. OMS. 2013:1-39.

2. WHO. GLOBAL STATUS REPORT on noncommunicable diseases 2014: "Attaining the nine global noncommunicable diseases targets; a shared responsibility." WHO. 2014: 9-33.

3. Bener A, Kim EJ, Mutlu F, Eliyan A, Delghan H, Nofal E, et al. Burden of diabetes mellitus attributable to demographic levels in Qatar: an emerging public health problem. Diabetes Metab Syndr. 2014; 8(4): 216-220. DOI: 10.1016/j.dsx.2014.09.005.

4. American Diabetes Association. Standards of medical care in Diabetes - 2016. Diabetes Care. 2016; 39(suppl 1): s6-s12. DOI: 10.2337/dc16-S003.

5. Ali MK, Bullard KM, Saaddine JB, Cowie CC, Imperatore G, Gregg EW. Achievement of goals in U.S. diabetes care, 1999-2010. N Engl J Med. 2013; 368(17): 1613-1624. DOI: 10.1056/ NEJMsa1213829.

6. Sandy R, Connor U. Variation in medication adherence across patient behavioral segments: a multi-country study in hypertension. Patient Prefer Adherence. 2015; 9: 539-1548. DOI: 10.2147/PPA.S91284.

7. Chang PY, Chien LN, Lin YF, Chiou HY, Chiu WT. Nonadherence of oral antihyperglycemic medication will increase risk of end-stage renal disease. Medicine (Baltimore). 2015; 94(47): 1-8. DOI: 10.1097/MD.0000000000002051.

8. Néboa Z, Renata V, Álvaro H, Juan O, Marta R. Guía Metodológica Para Estimar Los Costos Asociados a La Diabetes. (Instituto Max Weber, ed.). Madrid; 2015.

9. Dobbels F, Berben L, De Geest S, Drent G, Lennerling A, Whittaker C, et al. The psychometric properties and practicability of self- report instruments to identify medication nonadherence in adult transplant patients: a systematic review. Transplantation. 2010; 90(2): 205-219. DOI: 10.1097/TP.0b013e3181e346cd.

10. Stirratt MJ, Dunbar-Jacob J, Crane HM, Simoni JM, Czajkowski S, Hilliard ME, et al. Self-report measures of medication adherence behavior: recommendations on optimal use. Transl Behav Med. 2015; 5(4): 470482. DOI: 10.1007/s13142-015-0315-2.

11. López Romero LA, Romero Guevara SL, Parra DI, Rojas Sánchez LZ. Adherencia al tratamiento: Concepto y medición. Hacia Promoc salud. 2016; 21(1): 117-137. DOI: 10.17151/hpsal.2016.21.1.10.

12. Organización Mundial de la Salud (OMS). Adherencia a los tratamientos a largo plazo: pruebas para la acción. OMS-OPS. 2004: 127-132.

13. Sue Moorhead MJ. Clasificación de Resultados de Enfermería (NOC). Cuarta Edi. Elsevier Health 
Sciences; 2009.

14. Romero Guevara S, Parra D, Lyda Zoraya R, Orozco O. Validación de la etiqueta de resultado de enfermería para la medición de la adherencia al tratamiento. In: II Congreso Latinoamericano NANDA-Internacional; 2015.

15. Charlson ME, Pompei P, Ales KL, MacKenzie CR. A new method of classifying prognostic comorbidity in longitudinal studies: development and validation. J Chronic Dis. 1987; 40(5): 373-383. DOI: 10.1016/0021-9681(87)90171-8.

16. D'Agostino RB, Vasan RS, Pencina MJ, Wolf PA, Cobain M, Massaro JM, et al. General cardiovascular risk profile for use in primary care: the framingham heart study. Circulation. 2008; 117(6): 743-753. DOI: 10.1161/CIRCULATIONAHA.107.699579.

17. Natarajan N, Putnam W, Van Aarsen K, Lawson B, Burge $F$. Adherence to antihypertensive medications among family practice patients with diabetes mellitus and hypertension. Can Fam Physician. 2013; 59(2): 93-100. DOI: 59/2/e93 [pii].

18. Fletcher BR, Hartmann-Boyce J, Hinton L, Mcmanus RJ. The effect of self-monitoring of blood pressure on medication adherence and lifestyle factors: a systematic review and meta-analysis. Am J Hypertens. 2015; 28(10): 1209-1221. DOI: 10.1093/ajh/hpv008.

19. García Pérez AM, Leiva Fernández F, Martos Crespo F, García Ruiz AJ, Prados Torres D, Sánchez de la Cuesta y Alarcón F. ¿Cómo diagnosticar el cumplimiento terapéutico en atención primaria ? Med Fam. 2000; 1: 13-19.

20. Thinyane KH, Mothebe T, Sooro M, Namole LD, Cooper V. An observational study of hypertension treatment and patient outcomes in a primary care setting. Pan Afr Med J. 2015; 8688: 1-10. DOI: 10.11604/pamj.2015.20.424.5040.

21. Migneault JP, Dedier JJ, Wright JA, Heeren T, Campbell MK, Morisky DE, et al. A culturally adapted telecommunication system to improve physical activity, diet quality, and medication adherence among hypertensive African-Americans: a randomized Controlled Trial. Ann Behav Med. 2012; 43: 62-73. DOI: 10.1007/s12160-011-9319-4.

22. Perk J, De Backer G, Gohlke H, Graham I, Reiner $Z$, Verschuren $\mathrm{M}$, et al. European Guidelines on cardiovascular disease prevention in clinical practice (version 2012). The Fifth Joint Task Force of the European Society of Cardiology and Other Societies on Cardiovascular Disease Prevention in Clinical Practice (constituted by representatives of nine societies and by invited experts). Eur Heart J. 2012; 33(13): 1635-1701. DOI: 10.1093/eurheartj/ehs092.
23. van Bruggen R, Gorter KJ, Stolk RP, Verhoeven RP, Rutten GE. Implementation of locally adapted guidelines on type 2 diabetes. Fam Pract. 2008; 25(6): 430-437. DOI: 10.1093/fampra/cmn045.

24. McGill M. La necesidad de tacto, franqueza y honestidad al hablar sobre las complicaciones. Diabetes Voice. 2007; 52: 29-32.

25. da Silva Barreto M, Oliveira Reiners AA, Marcon SS. Conocimiento sobre hipertensión arterial y factores asociados a la no adhesión a la farmacoterapia. Rev Lat Am Enferm. 2014; 22(3): 491-498. DOI:10.1590/0104-1169.3447.2442.

26. Ma C. A cross-sectional survey of medication adherence and associated factors for rural patients with hypertension. Appl Nurs Res. 2016; 31: 94-99. DOI: 10.1016/j.apnr.2016.01.004.

27. Ahn YH, Ham OK. Factors associated with medication adherence among medicalaid beneficiaries with hypertension. West $\mathrm{J}$ Nurs Res. 2016: 38(10): 1298-1312. DOI: 10.1177/0193945916651824.

28. Boima V, Ademola AD, Odusola AO, Agyekum F, Nwafor CE, Cole H, et al. Factors associated with medication nonadherence among hypertensives in Ghana and Nigeria. Int J Hypertens. 2015; 2015: 205716. DOI: 10.1155/2015/205716.

29. Nazir SU, Hassali MA, Saleem F, Bashir S, Aljadhey H. Disease related knowledge, medication adherence and glycaemic control among patients with type 2 diabetes mellitus in Pakistan. Prim Care Diabetes. 2016; 10(2): 136-141. DOI: 10.1016/j. pcd.2015.09.004.

30. Magnabosco P, Calavari Teraoka E, Meirelles de Oliveira E, Aparecida Felipe E, Freitas D, MarchiAlves LM. Análisis comparativa de la no adhesión al tratamiento medicamentoso de la hipertensión arterial sistémica en población urbana y rural 1 . Rev Lat Am Enferm. 2015; 23(1): 20-27. DOI: 10.1590/0104-1169.0144.2520.

31. Yassine M, Al-Hajje A, Awada S, Rachidi S, Zein S, Bawab W, et al. Evaluation of medication adherence in Lebanese hypertensive patients. J Epidemiol Glob Health. 2016; 6(3): 157:167. DOI: 10.1016/j. jegh.2015.07.002.

32. Hashmi SK, Afridi MB, Abbas K, Sajwani DS, Saleheen D, Frossard P, et al. Factors associated with adherence to anti-hypertensive treatment in Pakistan. Plos One. 2007; 2(3). e280. DOI: 10.1371/ journal.pone.0000280.

33. Al-ramahi R. Adherence to medications and associated factors : A cross-sectional study among Palestinian hypertensive patients. J Epidemiol Glob Health. 2015; 5(2): 125-132. DOI: 10.1016/j.jegh.2014.05.005. 